\title{
Hypothesis testing of genetic similarity based on RAPD data using Mantel tests and model matrices
}

\author{
Flávia Melo Rodrigues ${ }^{1}$, José Alexandre Felizola Diniz-Filho ${ }^{2}$, Luiz Artur Mendes Bataus ${ }^{3}$ \\ and Rogério Pereira Bastos ${ }^{2}$ \\ ${ }^{1}$ Programa de Pós-Graduação em Biologia (Genética), ICB, Universidade Federal de Goiás, Goiânia, \\ GO, Brazil. \\ ${ }^{2}$ Departamento de Biologia Geral, ICB, Universidade Federal de Goiás, Goiânia, GO, Brazil. \\ ${ }^{3}$ Departamento de Ciências Fisiológicas, ICB, Universidade Federal de Goiás, Goiânia, GO, Brazil.
}

\begin{abstract}
Clustering and ordination procedures in multivariate analyses have been widely used to describe patterns of genetic distances. However, in some cases, such as when dealing with Jaccard coefficients based on RAPD data, these techniques may fail to represent genetic distances because of the high dimensionality of the genetic distances caused by stochastic variation in DNA fragments among the units analyzed (species or populations). In this note, we show how Mantel tests can be used to test hypotheses about genetic distances and avoid problems with multivariate data representation. The procedure is illustrated with RAPD data comparing 20 anuran species from the cerrado, in which Jaccard genetic distances were compared to three model matrices linking the species at distinct taxonomic levels.
\end{abstract}

Key words: Anura, clustering, genetic distances, Mantel test, RAPD.

Received: January 24, 2001; accepted: November 4, 2002.

\section{Introduction}

The multivariate analysis of genetic distances in population genetics and molecular systematics has been one of the most important tools for recovering spatial or taxonomic structure in data (Lessa, 1990; Swofford et al., 1996; Meyer, 1997). Among the many analytical procedures available, the unweighted pair-group method using arithmetic averages (UPGMA) and, more recently, the neighbor-joining (NJ) clustering algorithms, as well as some ordination techniques, such as principal coordinates (PCORD) and non-metric multidimensional scaling (NMDS), have been widely used to describe genetic distances and to assess the structure of genetic data in a reduced dimensional space.

When dealing with multivariate analyses, it is always desirable to verify the distortions in the output caused by the dimensional reduction process, and to determine whether the representation of genetic distances in one, two or three dimensions is in fact informative. In UPGMA clustering, the most commonly used measure of this distortion is the cophenetic correlation coefficient, which measures

Send correspondence to José Alexandre Felizola Diniz-Filho. Departamento de Biologia Geral, ICB, Universidade Federal de Goiás, CP 131, 74.001-970 Goiânia, GO, Brasil. E-mail: diniz@ @icb1.ufg.br. the correspondence between the original genetic distances among samples and the distances defined along the phenogram (Sneath and Sokal, 1973; Rodrigues and Diniz-Filho, 1998). A similar approach can be used to evaluate the results from ordination displays, although in this case there are also other specific measures of distortion, such as the accumulated explanatory power of the eigenvalues in PCORD or the "stress" value (S) in NMDS (Johnson and Wichern 1992).

Different microevolutionary processes acting at the populational level, especially those generating clinal or reticulated patterns of genetic variation across geographic space, always produce patterns of population differentiation that are difficult to represent in a few dimensions by clustering or ordination techniques (Lessa, 1990; Rodrigues and Diniz-Filho, 1998). Stochastic evolutionary processes at higher taxonomic levels, such as independent evolution by Brownian motion of quantitative characters or gene frequencies (Hansen and Martins, 1996; Diniz-Filho, 2000), generate a similar difficulty in representing genetic distances among species. Another problem in using these multivariate procedures is that they are usually applied in an exploratory way, without a formal statistical test of the hypotheses about the relationships among populations or species (Neff and Marcus, 1980). The main problem with 
standard statistical tests used to compare genetic distances directly is that the elements of genetic distance matrices are not independent, such that Type I errors are inflated to an unknown degree (Manly, 1997).

Despite these problems, in most cases the cophenetic correlations from UPGMA clustering of genetic distances, based on isozyme data from local populations, are elevated (Rodrigues and Diniz-Filho, 1998). However, using random amplified polymorphic DNA (RAPD) to assess genetic variability may be slightly more complicated compared to isozymes since the nature of the data may generate a large amount of random variation, especially at higher taxonomic levels (Hillis et al., 1996). More seriously, many papers deal with individuals (and not with allele frequencies in local populations or samples) and try to cluster or ordinate them (see for example Colombo et al., 2000). Although careful inspection of the results could eliminate part of this random noise (such as choosing only the stronger, repeatable bands) (see Dominguez, 1998), this is usually a subjective procedure and does not necessarily avoid the problems of high dimensionality in the genetic structure itself.

In these cases, the association or correlation between the variables used (frequencies or presence/absence of bands) is low and, consequently, the Jaccard coefficients commonly used to estimate genetic similarity among individuals are defined in a genetic space with a very high number of orthogonal dimensions. Thus, despite the usefulness of RAPD in assessing patterns of genetic divergence among local populations (Swofford et al., 1996; Sunnucks, 2000; Russel et al., 2002), the clustering and ordination techniques commonly used may fail to accurately represent genetic distances, especially when dealing with Jaccard coefficients calculated among individuals. This problem can often be detected as a low cophenetic correlation coefficient, a low support of many clusters following bootstrapping of the genetic distances, and low relative eigenvalues of the ordination axes (see Lessa, 1990; Rodrigues and Diniz-Filho, 1998).

Mantel tests have been widely used to evaluate patterns of genetic distance, especially to test the statistical significance of matrix correlation between genetic and geographic distances (Smouse et al., 1986; Manly, 1985, 1997; Diniz-Filho, 1998, 2000). In this note, we show how Mantel tests can be used in a more general way to test different hypotheses about genetic distances. This procedure can be very useful when dealing especially with RAPD data in which the elevated number of dimensions makes the visual inspection of patterns difficult and complicates the testing of hypotheses using standard clustering and ordination techniques.

\section{Mantel tests and model matrices}

The Mantel test $(Z)$ of matrix correspondence is given by:

$$
\mathrm{Z}=\Sigma_{\mathrm{i}} \Sigma_{\mathrm{j}}\left(\mathrm{E}_{\mathrm{ij}} \mathrm{M}_{\mathrm{ij}}\right)
$$

Where $\mathrm{E}_{\mathrm{ij}}$ and $\mathrm{M}_{\mathrm{ij}}$ are the $i$ th and $j$ th elements of the square symmetric matrices $\mathbf{M}$ and $\mathbf{E}$ being compared. The statistical significance of this association is measured by a randomization procedure in which the order of the elements in one of these matrices is randomly permutated several times and a Z-value is calculated for each permutation (Manly, 1985, 1997; Diniz-Filho, 1998).

Because the Z-value of the Mantel test is only a sum of products between the elements of the matrices, its value depends on the number of samples and on the magnitude of the numbers in the matrices (different scales of geographic distances, for example, that can be measured in meters or kilometers). However, it is possible to show that this $\mathrm{Z}$-value is in fact the non-centered numerator of the Pearson product-moment correlation coefficient between the elements of $\mathbf{M}$ and $\mathbf{E}$ (Manly, 1985). Since the means and variances of these elements remain unchanged with the permutations, the Z-value and the Pearson correlation maintain a monotonic relationship. In other words, the Mantel test, by randomization of the $\mathrm{Z}$-values, is a procedure to test the statistical significance of the matrix correlation coefficient. The advantage of using the matrix correlation instead of Z-value as a descriptor of the relationship is that it varies between -1.0 and 1.0, such that the strength of the association between the two matrices is easier to interpret.

Most applications of the Mantel test and matrix correlation deal with continuous distance or similarity values, such as pairwise genetic, geographic or environmental distances between local populations (Diniz-Filho, 1998, 2000; Telles et al. 2001). However, it is possible to compare the genetic distances with discrete matrices in which the elements are one or zero, indicating that samples are "linked" or not, respectively. The pattern of links established in one of the matrices, which we term here the "model matrix", allows one to test different hypotheses about structure in genetic distances.

For example, linking pairs of local populations at increasing geographic distances (thereby generating distinct model matrices) allows one to test whether the matrix correlation decreases with increasing geographic distance, and yields a multivariate correlogram in a multivariate spatial autocorrelation analysis (Sokal et al., 1986; Diniz-Filho, 1998). These model matrices can also be useful for testing more specific hypotheses, including pathways of gene flow, adaptive hypotheses with phenotypic patterns (such as when dealing with problems of morphological integration - see Cheverud et al., 1989) and taxonomic or biogeographic structure in data (for example, testing whether samples before and after the introduction of an ecological or geographical barrier are different). When dealing with genetic variation among individuals sampled at distinct localities, the Mantel test works as a one-way 
analysis of variance (ANOVA) (a completely random design), and tests by randomization if pairwise genetic distances between individuals within a locality are smaller than the genetic distances between individuals at different localities. In other words, one can test whether localities are genetically different, is a manner analogous to one-way analysis of molecular variance (AMOVA).

There are two main advantages of this general approach using the Mantel test, when compared to clustering and ordination procedures: 1) Hypotheses can be tested statistically instead of by visual inspection of the genetic distances in a phenogram or reduced dimensional space, and 2) this procedure uses all information in the genetic distances, making it less sensitive to the distortions caused by dimensional reduction. Consequently, this approach is still useful when dealing with high-dimensional systems that cannot be represented in a few ordination axes.

\section{Genetic divergence among Anuran species assessed by RAPD}

To illustrate the procedures described above, we analyzed the RAPD data of 20 species of frogs collected in central Brazil. Specimens were collected at two sites in the state of Goiás (Florestal Experimental Station - EFLEX, in the municipality of Silvânia and on "Lagoa Grande" farm, in the municipality of Pontalina). The species were distributed in nine genera and three families (Hylidae, Leptodactylidae and Bufonidae). Although the RAPD technique is better applied to compare local populations or closely related species rather than distant species as done here (Swofford et al., 1996; Sunnucks, 2000), the high level of polymorphism obtained in this dataset strongly inflated the number of dimensions in the genetic similarity and thus furnished a very useful example of how to use the Mantel test with model matrices. Data obtained at the populational level may have the same problems, especially when analyzing many primers and fragments in individuals from differ- ent populations (see Colombo et al., 2000, for a recent example).

DNA was isolated from blood and liver using the extraction procedures described by Sambrook et al. (2001), with a few modifications. The PCRs were done in a $20 \mu \mathrm{L}$ reaction volume containing $30 \mathrm{ng}$ of genomic DNA, $10 \mathrm{ng}$ of primer, 1.5 units of Taq DNA polymerase (Pharmacia), $2.5 \mathrm{mM}$ of each dNTP and $10 \mathrm{mM}$ Tris-HCl, $\mathrm{pH} 8.0$, $50 \mathrm{mM} \mathrm{KCl}$ and $2.5 \mathrm{mM} \mathrm{MgCl}_{2}$. Fifty arbitrary primers were tested, of which nine were chosen because of their amplification pattern the quantity of fragments produced, their quality and their reproducibility for all species. DNA was amplified in a thermocycler (PTC-100 MJ Research) programmed as follows: $94^{\circ} \mathrm{C}$ for $5 \mathrm{~min}$, followed by $40 \mathrm{cy}-$ cles of $30 \mathrm{~s}$ at $94{ }^{\circ} \mathrm{C}, 1 \mathrm{~min}$ at $35^{\circ} \mathrm{C}$ and $1 \mathrm{~min}$ at $72^{\circ} \mathrm{C}$, with a final stage of $3 \mathrm{~min}$ at $72{ }^{\circ} \mathrm{C}$, and then maintained at $4{ }^{\circ} \mathrm{C}$ prior to analysis. The amplification products were analyzed by electrophoresis in $1.5 \%$ agarose gels, stained with ethidium bromide $(0.5 \mu \mathrm{g} / \mathrm{mL})$. Fragments were visualized under UV light and photographed for further codification. Only strong, reproducible bands were used.

A total of 133 fragments were obtained from the nine primers and coded as one or zero to indicate their presence or absence in each species, respectively. All of the fragments were polymorphic, with a size ranging from 100 to $1700 \mathrm{bp}$. The number of fragments per primer varied from 7 to 24 (Table I). The Jaccard coefficients were used to establish a pairwise genetic similarity matrix between individuals (i.e., species) that was initially analyzed by UPGMA clustering and different ordination procedures using version 1.5 of the software NTSYS-Pc (Rohlf, 1989).

All standard clustering and ordination procedures failed to produce an adequate representation of the genetic similarity. The cophenetic correlation of the UPGMA was 0.680 , a relatively low value by standard criterion (usually only values above 0.8 provide a reasonable representation of similarity). The explanation power of the first two eigen-

Table I - Matrix correlations (r) and their Type I errors (P) obtained using Mantel tests with 5000 random permutations compare of genetic similarities (Jaccard coefficient) based individual primer and overall RAPD data in each model matrix expresses a distinct relationship model for the species.

\begin{tabular}{|c|c|c|c|c|c|c|}
\hline \multirow[t]{2}{*}{ Primer } & \multicolumn{2}{|c|}{ Model I } & \multicolumn{2}{|c|}{ Model II } & \multicolumn{2}{|c|}{ Model III } \\
\hline & $\mathrm{R}$ & $\mathrm{P}$ & $\mathrm{r}$ & $\mathrm{P}$ & $\mathrm{r}$ & $\mathrm{P}$ \\
\hline 62 & 0.151 & 0.037 & 0.128 & 0.0620 & 0.160 & 0.027 \\
\hline 63 & 0.043 & 0.301 & 0.021 & 0.3504 & 0.035 & 0.267 \\
\hline 64 & 0.029 & 0.382 & 0.064 & 0.1950 & 0.059 & 0.212 \\
\hline OPA-01 & 0.063 & 0.421 & 0.040 & 0.1026 & 0.00002 & 0.346 \\
\hline OPA-03 & 0.227 & 0.018 & 0.148 & 0.0470 & 0.209 & 0.008 \\
\hline OPC-02 & 0.092 & 0.111 & 0.023 & 0.3370 & 0.059 & 0.197 \\
\hline OPC-15 & 0.077 & 0.164 & 0.030 & 0.3670 & 0.013 & 0.375 \\
\hline OPH-11 & 0.067 & 0.209 & 0.101 & 0.1116 & 0.103 & 0.102 \\
\hline OPH-14 & 0.044 & 0.426 & 0.014 & 0.4490 & 0.031 & 0.471 \\
\hline All primers & 0.224 & 0.003 & 0.082 & 0.1304 & 0.161 & 0.018 \\
\hline
\end{tabular}


values obtained using principal coordinate analysis was only $16.8 \%$, and indeed both eigenvalues were lower than null expectation under a broken-stick distribution (see Jackson, 1993). These results indicated a large amount of random variation in the data and a poor structure, such that visual inspection for patterns world have been useless. Even representin the Jaccard coefficients using non-metric multidimensional scaling (NMDS), a powerful non-linear ordination technique, failed, with a stress value equal to 0.697 and 0.629 , after optimizing the ordination solution in two and three dimensions, respectively. In NMDS, only stress values less than 0.01 are considered adequate (Johnson and Wichern, 1992), and this low value cannot be achieved even when representing data in five dimensions (the maximum capacity for NMDS in NTSYS 1.5).

Before abandoning the data as useless for revealing any relationship among species, we sought to develope hypotheses of relatedness among these species and to create model matrices to represent them. The most obvious model of relationships was the standard taxonomic classification of the species, which at least partly reflect evolutionary patterns over time. We first created a model matrix (Model I) with a value of one if a given pair of species belonged to the same genus and zero elsewhere. We used the same reasoning to create another model matrix representing the connections among species in terms of their families (Model II). Finally, a third matrix Model III was constructed by combining the two previous matrices, in which a value of 1.0 was assigned to pairs of species in the same genus, 0.5 was assigned to species in different genera but in the same family, and zero indicated that a the pair of species being compared belonged to different families. The three relationships expressed by the model matrices are shown in Figure 1.

Mantel tests were then used to assess the statistical significance of the matrix correlation between the Jaccard similarity matrix and the model matrices. Statistical significance was tested using 5,000 random permutations. To determine which primer contributed most to the overall genetic structure, we also constructed different Jaccard similarity matrices using the fragments obtained from each primer (Table I). Primers 062 and OPA-03 tended to differentiate species in different genera and families, although the ability to discriminate as revealed by the squared correlation coefficient $\left(\mathrm{R}^{2}\right)$, was usually low, indicating a large amount of random variation. The $\mathrm{R}^{2}$ values, which were usually very low (frequently less than $5 \%$ ), were equivalent to the intraclass correlations in ANOVA (Sokal and Rohlf, 1995), indicating that the relative amount of variation among groups (taxa) was very low compared to unique variation at the species level.

One interesting aspect of these analyses was that the accumulation of the two taxonomic levels used to create Model III, did not increase the magnitude of $\mathrm{R}^{2}$ when compared to Models I and II, indicating that the two levels were
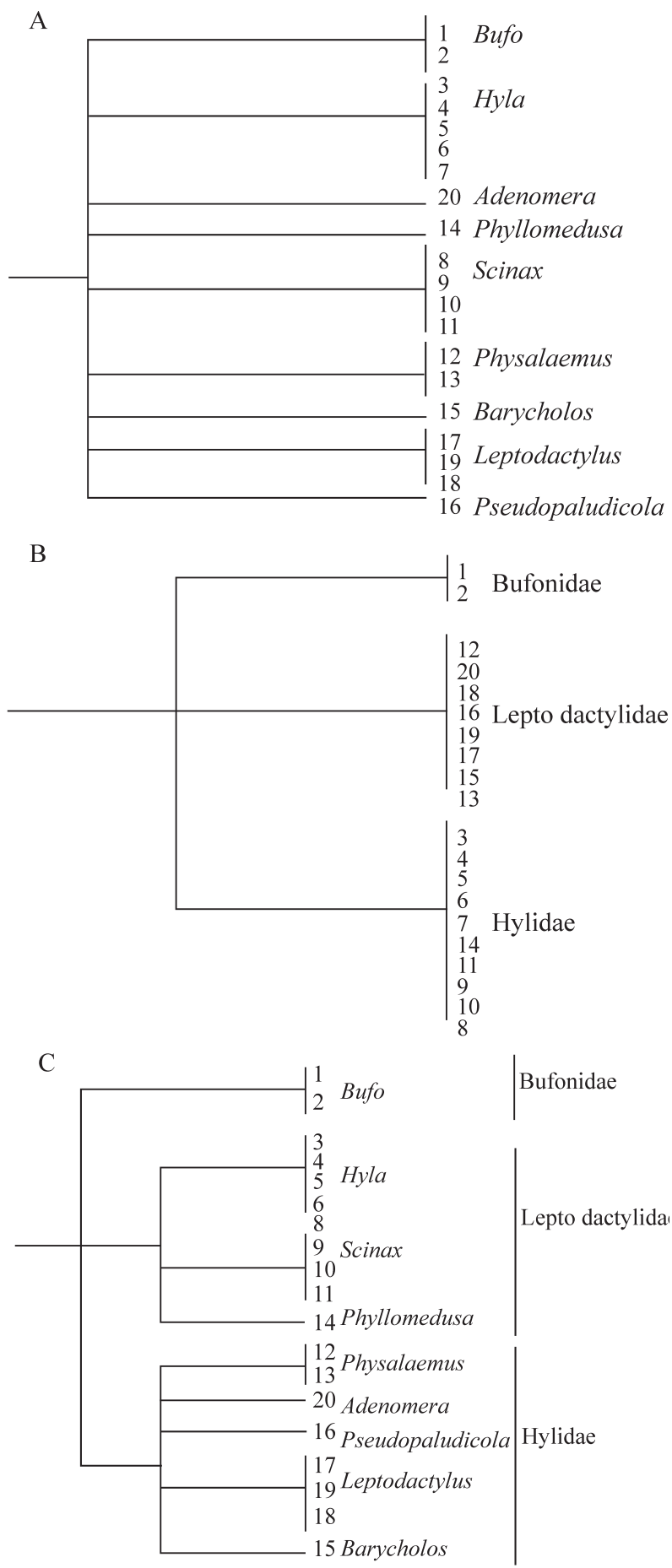

Figure 1 - Relationship among 20 species of anurans from the cerrado region under the three models of taxonomic relationship, linking species within the same genera (A), species within the same family (B) and combining the two hierarchical levels (C). These matrices were compared with the divergence matrix using the Jaccard coefficient end the Mantel test with 5000 random permutations.

not additive in the expression of the genetic variability. Indeed, the species seemed to be almost independent for these data and the hierarchical structure at the genus and family levels had only a small effect when compared to the varia- 
tion within each lineage. The high level of polymorphism indicated that the species are very different in their RAPD markers and more or less equidistant in the hyperspace created by these data. This probably occurred because the species diverged a long time ago, such that common fragments of RAPD were lost by random processes and any phylogenetic signal was destroyed. In agreement, with this, paleontological and molecular data indicate that two of the families analyzed here diverged more than 50 million of years ago (Hay et al., 1995; Hedges and Maxson, 1993).

Despite the difficulties in using RAPD data to establish phylogenetic or taxonomic relationships in the example of very distantly related species used here, the main purpose of this paper was to describe alternative multivariate procedures for analyzing highly dimensional genetic distances, especially when these are based on individuals. This approach may be used to select only more informative primers for further clustering and ordination analyses. Associating the Mantel test with model matrices allows one to explore alternative hypotheses of the genetic relationship among individuals using explicit statistical tests and not simply visual inspection of the results from multivariate analyses.

\section{Acknowledgments}

The authors thank Leandro R. Monteiro and Aparecido Divino da Cruz for helpful discussions and suggestions to previous versions of this work, and Mariana Pires de Campos Telles for critical reading of previous versions of the manuscript. Financial support was provide by CAPES (master's degree scholarship to F.M.R.), CNPq (research fellowships to J.A.F.D.F. and R.P.B. and PCOPG 520804/99-6) and FUNAPE/UFG (grant to F.M.R.).

\section{References}

Cheverud JM, Wagner GP, Dow MM (1989) Methods for the comparative analysis of variation patterns. Syst Zool 38:201-213.

Colombo C, Second G and Charrier A (2000) Diversity within American cassava germ plasm based on RAPD markers. Genet Mol Biol 23:189-200.

Diniz-Filho JAF (1998) Análise de autocorrelação espacial: inferências microevolutivas e aplicações em genética de populações. In: Duarte FAM (ed) Série Monografias 6, Sociedade Brasileira de Genética, Ribeirão Preto, pp 83-145.

Diniz-Filho JAF (2000) Métodos Filogenéticos Comparativos. Holos, Ribeirão Preto, 162 pp.

Dominguez PA (1998) An evaluation of RAPD fragment reproductibility and nature. Mol Ecol 7:1347-1357.

Hansen TF and Martins EP (1996) Translating between microevolutionary process and macroevolutionary patterns: the correlation structure of interspecific data. Evolution 50:1404-1417.

Hay JM, Ruvinsky H, Hedges SB and Maxson LR (1995) Phylogenetic relationships of amphibian families inferred from
DNA sequences of mitochondrial $12 \mathrm{~S}$ and $16 \mathrm{~S}$ ribosomal RNA genes. Mol Biol Evol 12:928-937.

Hedges SB and LR Maxson (1993) A molecular perspective on lissamphibian phylogeny. Herpet Monogr 7:42-45.

Hillis DM, Mable BK and Moritz C (1996) Applications of molecular systematics: the state of the field and a look to the future. In: Hillis DM, Moritz C, Marble BK (eds) Molecular Systematics. 2nd ed. Sinauer Press, Sunderland, Massachusetts, pp 515-543.

Jackson DA (1993) Stopping rules in principal component analysis: a comparison of heuristic and statistical approaches. Ecology 74:2204-2214.

Johnson RA and Wichern DW (1992) Applied Multivariate Statistical Analysis. 3nd. ed. Chapman and Hall, London, $642 \mathrm{pp}$.

Lessa E (1990) Multidimensional analysis of geographic genetic structure. Syst Zool 39:242-252.

Manly BFJ (1985) The Statistics of Natural Selection. Chapman and Hall, London, 484 pp.

Manly BFJ (1997) Randomization, Bootstrap and Monte Carlo Methods in Biology. Chapman and Hall, London, 424 pp.

Meyer D (1997) Análise filogenética de seqüências de DNA. In: Amorim DS (ed) Elementos Básicos de Sistemática Filogenética. Holos, Ribeirão Preto, pp 187-212.

Neff NA and Marcus LF (1980) A Survey of Multivariate Methods for Systematics. Privately published, New York, $234 \mathrm{pp}$.

Rodrigues FM and Diniz-Filho JAF (1998) Hierarchical structure of distances: effects of matrix size, spatial distribution and correlation structure among gene frequencies. Genet Mol Biol 21:233-240.

Rohlf FJ (1989) NTSYS: Numerical Taxonomy and Multivariate Analysis System. Exeter softwares, New York.

Russel JD, Waycott M, Chappill JA and James SH (2002) Molecular phylogenetic analysis of the evolution of complex hibridity in Isotoma petraea. Evolution 56:1296-1302.

Sambrook J, Fritsch EF and Maniatis T (1989) Molecular Cloning: a Laboratory Manual. 2nd. ed. Cold Spring Harbor Laboratory Press, New York, 999 pp.

Smouse PE, Long JC and Sokal RR (1986) Multiple regression and correlation extensions of the Mantel test of matrix correspondence. Syst Zool 35:627-632.

Sneath PHA and Sokal RR (1973) Numerical Taxonomy. W.H. Freeman, San Francisco, 576 pp.

Sokal RR and Rohlf FJ (1995) Biometry, 3rd. ed. W.H. Freeman, New York, 887 pp.

Sokal RR, Smouse PE and Neel JV (1986) The genetic structure of a tribal population, the Yanomama indians. XV. Patterns inferred by autocorrelation analysis. Genetics 114:259-287.

Sunnucks P (2000) Efficient genetic markers for population biology. Trends Ecol Evol 15:199-203.

Swofford DL, Olsen GJ, Waddell PJ and Hillis DM (1996) Phylogeny inference. In: Hillis DM, Moritz C and Marble BK (eds) Molecular Systematics. Sinauer Press, Sunderland, Massachusetts, pp 407-514.

Telles MPC, Silva RSM, Chaves LJ, Coelho ASG and DinizFilho JAF (2001) Divergência entre subpopulações de cagaiteira (Eugenia dysenterica) em resposta a padrões edáficos e distribuição espacial. Pesq Agrop Bras 36:13871394. 